\title{
Desarrollo de un método para evaluar el daño ocasionado por aves en cultivos comerciales de soja recién emergida
}

\author{
Development of a method for assessing damage caused by birds \\ in sprouting soybean in commercial crops \\ Nadia Bouㄴ, Sebastián Dardanelli², Lourdes Olivera ${ }^{3}$, Guillermo Tellechea ${ }^{3}$, \\ Laura Addy Orduna², Sonia Canavelli ${ }^{2}$ y Ethel Rodríguez ${ }^{4 *}$
}

\section{RESUMEN}

El cultivo de soja (Glycine max) es uno de los principales rubros productivos en Sudamérica. Algunas especies de aves pueden consumir los cotiledones de las plántulas de soja recién emergidas causando daño al cultivo. Con el objetivo de contar con estimaciones precisas del daño por aves en soja recién emergida se desarrolló un método de evaluación, suponiendo un gradiente de daño en función de la distancia del lote a parches con árboles usados como refugio por las aves. En base a estimaciones de media, desvío estándar, intervalo de confianza y varianza de los datos obtenidos en seis lotes del oeste de Uruguay se determinó un diseño y tamaño de muestra óptimo para minimizar el esfuerzo y tiempo de evaluación, manteniendo un alto nivel de precisión. Dicha metodología fue posteriormente aplicada en ocho lotes, obteniéndose un daño de 10 a $50 \%$ cerca de los árboles y de 1 a $5 \%$ en el resto del cultivo. El método propuesto brinda información objetiva del daño y constituye una herramienta útil para la toma de decisiones a nivel predial.

Palabras clave: diseño de muestreo, tamaño muestral óptimo, cuantificación del daño, daño por aves, Glycine max.

\begin{abstract}
Soybean (Glycine max) cultivation is one of the main commodities in South America. Some bird species can consume the cotyledons of soybean seedlings newly emerged, causing crop damage. In order to have accurate estimates of the bird damage in sprouting soybean, we developed an assessment method assuming a gradient of damage based on the distance from the crop to a patch of trees used as shelter by birds. Based on the mean, standard deviation, confidence interval and data variance, obtained in six soybean fields located in western Uruguay, we determined a sampling design and optimal sample size to minimize the effort and time evaluation, maintaining a high level of accuracy. We subsequently applied this methodology on eight soybean fields. The damage near trees was between 10 and 50\% while in the rest of the field was between 1 and $5 \%$. The proposed method provides objective information on losses occurred, constituting a useful tool for decision making at farm level.
\end{abstract}

Key words: sampling design, optimum sample size, quantification of damage, bird damage, Glycine max.

\section{Introducción}

El cultivo de soja o soya (Glycine max) en Sudamérica ha experimentado un crecimiento continuo en la superficie cultivada, convirtiéndose en uno de los principales rubros productivos de la región en menos de 10 años (Catacora-Vargas et al., 2012). Este cultivo constituye el mayor rubro de exportación en Paraguay, Brasil, Argentina y Uruguay, aportando aproximadamente el 50\% de la

1 Instituto de Investigaciones Biológicas Clemente Estable (IIBCE), Departamento de Biodiversidad y Genética, Av. Italia 3318, C.P. 11600 , Montevideo, Uruguay.

2 Instituto Nacional de Tecnología Agropecuaria (INTA), Estación Experimental Agropecuaria Paraná, Factores Bióticos y Protección Vegetal, Ruta 11 Km 12,7, Oro Verde C.P. 3101, Entre Ríos, Argentina.

3 Instituto Nacional de Investigación Agropecuaria (INIA), Estación Experimental La Estanzuela, Ruta 50, Km. 11, C.P. 70000, Colonia, Uruguay.

4 Ministerio de Ganadería, Agricultura y Pesca (MGAP), Dirección General de Servicios Agrícolas. Millán 4703, C.P. 12900, Montevideo, Uruguay.

* Autor por correspondencia: erodriguez@mgap.gub.uy

Fecha de Recepción: 26 Julio, 2016.

Fecha de Aceptación: 22 Octubre, 2016.

DOI: $10.4067 / S 0718-34292016005000036$ 
soja producida en el mundo (Catacora-Vargas et al., 2012; USDA, 2015). En estos países la superficie cultivada se ha incrementado en más de un $200 \%$, superando las 45 millones de hectáreas, con una producción de más de 130 millones de toneladas (Rocha and Villalobos, 2012).

Entre múltiples factores que pueden impactar negativamente el cultivo de soja se encuentra el daño ocasionado por animales silvestres, incluyendo aves, mamíferos e invertebrados (Calvi et al., 1976; Ribeiro et al., 2008). Dentro del grupo de las aves, las especies que probablemente tengan una mayor incidencia sobre los daños son la paloma torcaza (Zenaida auriculata), paloma picazuro o de monte (Patagioenas picazuro) y paloma manchada o de ala manchada ( $P$. maculosa), debido a sus elevadas densidades en los paisajes que incluyen cultivos de soja (Calamari et al., 2011, 2012). Las palomas afectan al cultivo durante la siembra y en la emergencia, ya que se alimentan de las semillas y los cotiledones de las plántulas de soja recién emergidas (Rodríguez and Zaccagnini, 1998; Dardanelli et al., 2011; Rodríguez et al., 2011). El estadio de vulnerabilidad del cultivo corresponde a los primeros cinco a siete días, antes que brote el primer par de hojas verdaderas (Olivera and Rodríguez, 2014). Una vez desarrolladas las primeras hojas, la pérdida de los cotiledones no produciría disminución significativa en la sobrevivencia de la planta ni en el rendimiento del cultivo (Coulter and Nafziger, 2008; Moscardi et al., 2012).

A pesar de la importancia que parecería tener este tipo de daño en determinadas circunstancias (regiones, años, épocas de siembra), hay escasas metodologías estandarizadas y validadas estadísticamente para evaluar la magnitud del daño por las aves en cultivos en emergencia. Generalmente esto conlleva a tomar decisiones fundadas en evaluaciones subjetivas que favorecen la implementación de medidas de manejo más costosas que el propio daño (Dolbeer, 1981; Otis, 1992; Dolbeer et al., 1994; Engeman, 2000). Contar con un método confiable de evaluación de daño por aves en soja recién emergida permitiría estimar de forma más objetiva la relación costobeneficio de la resiembra o de la aplicación de una técnica para disminuir el daño por aves.

El presente trabajo tiene por objetivo desarrollar una metodología para evaluar el daño provocado por aves en cultivos comerciales de soja recién emergida, considerando un gradiente espacial en el daño. Se plantea la hipótesis que el daño no será homogéneo dentro de cada lote de cultivo, sino que será mayor en los sectores próximos a vegetación arbórea en forma de montes o cortinas que estas aves utilizan como refugio. Poner a prueba esta hipótesis no solo permitirá realizar evaluaciones costo-beneficio de alternativas de manejo para disminuir los daños, sino que hará posible evaluar la necesidad de incluir un margen de pérdida por aves en las expectativas de cosecha, conceptualizándolo en base a resultados objetivos (Otis, 1992; Zaccagnini, 1998; Engeman, 2000).

\section{Materiales y Métodos}

El trabajo se desarrolló en los Departamentos Paysandú, Río Negro, Soriano, Colonia y San José de la República Oriental del Uruguay (Figura 1). El clima típico de la región es templado y húmedo, con una temperatura media en invierno de $12,1{ }^{\circ} \mathrm{C}$ y en verano de $23,5^{\circ} \mathrm{C}$. Las precipitaciones son homogéneas durante todo el año con un promedio de lluvias de $1.284 \mathrm{~mm}$ anuales (Dirección de Climatología y Documentación, 2014). El paisaje original estaba cubierto por parches de bosques y pastizales naturales, correspondientes a la ecorregión Sabanas Uruguayas (Olson et al., 2001). En la actualidad, la zona de estudio tiene un alto impacto antrópico, con una expansión del área destinada a cultivos extensivos y forestaciones (Reolón et al., 2015).

El estudio se realizó en dos etapas. La primera, el modelo preliminar, consistió en la evaluación del daño por aves en base a un alto esfuerzo de muestreo y análisis estadísticos destinados a comprender el patrón de daño y estimar el tamaño de muestra que asegure precisión y minimice el esfuerzo de muestreo. La segunda etapa, el modelo definitivo, implicó la implementación del diseño y tamaño de muestra óptimos determinados en la etapa anterior.

Como primera instancia se llevó a cabo un estudio a campo para diferenciar los daños ocasionados por aves (palomas torcazas, de monte y de ala manchada) de los ocasionados por otros agentes causales, como liebres, hormigas y daño mecánico. El daño de dichas aves se distingue porque la planta presenta uno o ambos cotiledones faltantes, o cotiledones partidos a la mitad con sus bordes lisos. También pueden comerse la semilla o arrancar la plántula por completo generando plantas faltantes; sin embargo, este tipo de daño no fue evaluado ya que hay otros animales que comen de 


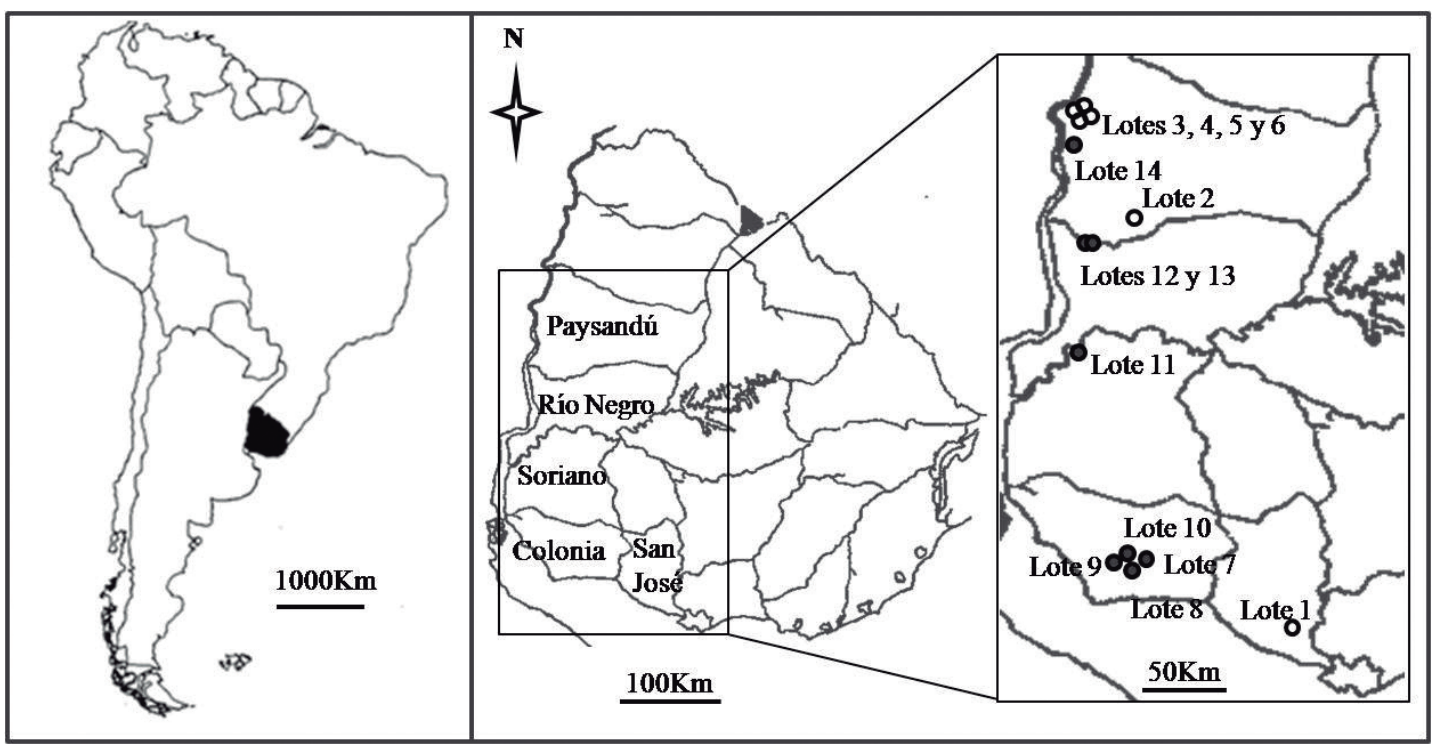

Figura 1. Ubicación geográfica de los lotes de soja muestreados en el litoral oeste de Uruguay. Círculos blancos: lotes evaluados en el modelo preliminar. Círculos grises: lotes evaluados en el modelo definitivo.

igual manera y porque la cantidad de semillas por metro lineal puede variar, por tanto es muy difícil saber dónde hay plantas faltantes y cuál es la causa de la misma. Previo a las evaluaciones de campo, los profesionales que registraron los datos fueron entrenados para discriminar el daño producido por aves del ocasionado por otros agentes causales.

La primera etapa del estudio, modelo preliminar, se llevó a cabo entre noviembre y diciembre del año 2012. En esta etapa se evaluó el daño por aves en seis lotes de soja comerciales de entre 25 y 49 ha del litoral oeste de Uruguay (Tabla 1). Esta evaluación se realizó mediante un muestreo estratificado en tres niveles: estrato, muestra y submuestra. Dado que las aves se refugian generalmente en los árboles y de allí descienden a alimentarse, las zonas del cultivo más cercanas a bosques y arboledas tienen mayores probabilidades de ser dañadas. En función de esto, el cultivo fue dividido en tres estratos a partir de un borde cercano a un parche de bosque, siendo el primero el más cercano al bosque y el tercero el más lejano. El primer estrato, "estrato borde", abarcó hasta $50 \mathrm{~m}$ de distancia del borde con árboles; el segundo, "estrato intermedio",

Tabla 1. Lotes de soja muestreados con ambos modelos.

\begin{tabular}{ccclc}
\hline Lotes de soja & Modelo & Departamento de Uruguay & Tipo de rastrojo & Superficie del lote (ha) \\
\hline Lote 1 & Preliminar & San José & Maíz & 25 \\
Lote 2 & Preliminar & Paysandú & Sorgo & 25 \\
Lote 3 & Preliminar & Paysandú & Sorgo & 49 \\
Lote 4 & Preliminar & Paysandú & Sorgo & 29 \\
Lote 5 & Preliminar & Paysandú & Sorgo & 40 \\
Lote 6 & Preliminar & Paysandú & Trigo & 25 \\
Lote 7 & Definitivo & Colonia & Avena & 25 \\
Lote 8 & Definitivo & Colonia & Avena y alfalfa & 40 \\
Lote 9 & Definitivo & Colonia & Avena & 20 \\
Lote 10 & Definitivo & Colonia & Avena & 30 \\
Lote 11 & Definitivo & Soriano & Sorgo & 40 \\
Lote 12 & Definitivo & Paysandú & Pastoreo & 10 \\
Lote 13 & Definitivo & Río Negro & Pastoreo & 60 \\
Lote 14 & Definitivo & Paysandú & Pastoreo & 25 \\
\hline
\end{tabular}


se ubicó entre 50 y 200 m; y el tercero, "estrato lejano", a más de 200 m completando el resto del lote. Dentro de cada estrato, se seleccionaron 10 surcos al azar, constituyendo cada surco una muestra. En cada surco se tomaron cinco submuestras de forma sistemática, ubicando la primera al azar y las siguientes a intervalos regulares (longitud surco/ $\mathrm{N}^{\circ}$ de submuestras). Cada submuestra consistió en 100 plantas consecutivas las que eran calificadas como sanas o dañadas (Figura 2). Se estimó el porcentaje de plantas dañadas por lote y por estrato (considerando el conjunto de todos los lotes), calculado a partir de la media con su error estándar. Para determinar diferencia significativa de daño entre estratos se utilizó el test no paramétrico de Kruskal-Wallis (debido a que no se cumplieron los supuestos de normalidad ni de homogeneidad de varianza), utilizando PAST 2.15 (Hammer et al., 2001). Para determinar el tamaño de muestra óptimo se calculó el error estándar para cada combinación de surco por submuestra y luego se graficó el error estándar en función del tamaño de la muestra, obteniéndose una pendiente negativa. El tamaño de muestra óptimo se determinó como el punto de inflexión donde la curva comenzó a hacerse constante a pesar del incremento del número de muestras. Este procedimiento fue realizado para cada estrato considerando el conjunto de lotes.

La segunda etapa, modelo definitivo, se implementó en ocho lotes de soja de entre 10 y 60 ha (Tabla 1) entre diciembre de 2012 y enero

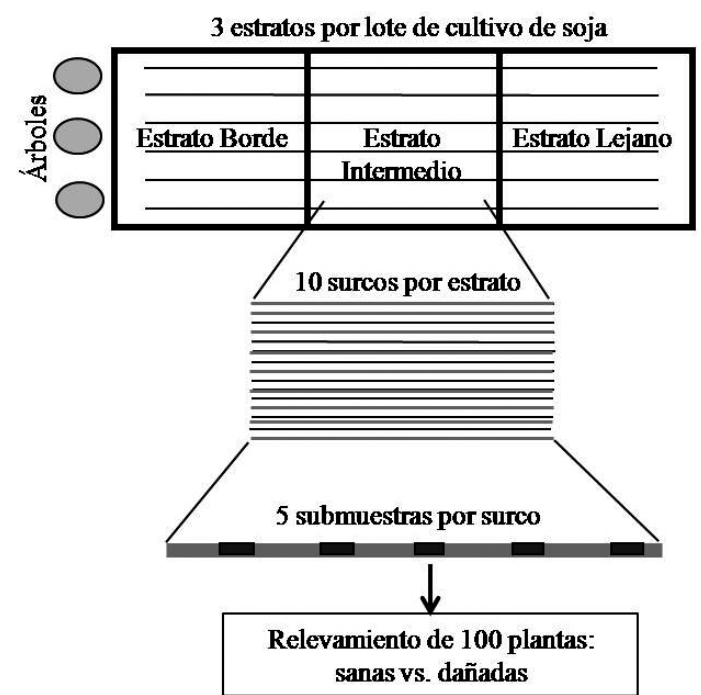

Figura 2. Modelo preliminar utilizado para evaluar el daño por aves en lotes de soja recién emergida. de 2013. Cada lote se dividió en un número de estratos determinado por presentar diferencia significativa en las medias de plantas dañadas en la etapa anterior. El número de surcos y submuestras se fijaron en función del tamaño de muestra óptimo definido en la etapa previa. En cada estrato dentro de cada lote se estimó la media, error estándar e intervalo de confianza. Para determinar diferencia significativa de daño entre estratos se utilizó el test no paramétrico de Mann Whitney, debido a que cada lote se dividió en dos estratos y no se cumplieron los supuestos de normalidad ni de homogeneidad de varianza. Asimismo, se registró el tiempo empleado en cada evaluación, para estimar el rango de tiempo promedio requerido para realizar la estimación de daño.

\section{Resultados y Discusión}

En el modelo preliminar, la media de plantas dañadas por aves en el estrato borde fue significativamente mayor (rango: 1,44-11,68\%) que en los estratos intermedio (rango: 1,06-5,60\%) y lejano (rango: $0,58-3,90 \%$, Figura 3), entre los que no se halló diferencia significativa. El error disminuyó más al aumentar el número de surcos que con el número de submuestras (Figura 4). Observando el punto donde la curva se vuelve constante se determinó que el tamaño óptimo de muestra (aquel que minimiza el error estándar con el mínimo esfuerzo) fue de 10 muestras con una única submuestra para el estrato borde, y tres muestras con una única submuestra para los de baja probabilidad de daño (estratos intermedio y lejano) (Figura 4). Con este diseño, todos los estratos alcanzaron aproximadamente el mismo nivel de error estándar (0,8-1). En base a estos resultados el modelo definitivo consistió en dividir el lote en dos estratos: uno de alta probabilidad de daño, ubicado cercano a una formación arbórea (equivalente al estrato borde) y otro de baja probabilidad de daño (equivalente al conjunto de estratos intermedio y lejano). Se consideraron 50 metros desde el borde colindante con árboles como límite entre ambos estratos. En el estrato de alta probabilidad de daño se evaluaron diez surcos (muestras), mientras en el estrato de baja probabilidad de daño se evaluaron tres, tomando en todos los casos una única submuestra de 100 plantas consecutivas por surco y evaluando cada una de ellas como sana o dañada por palomas 


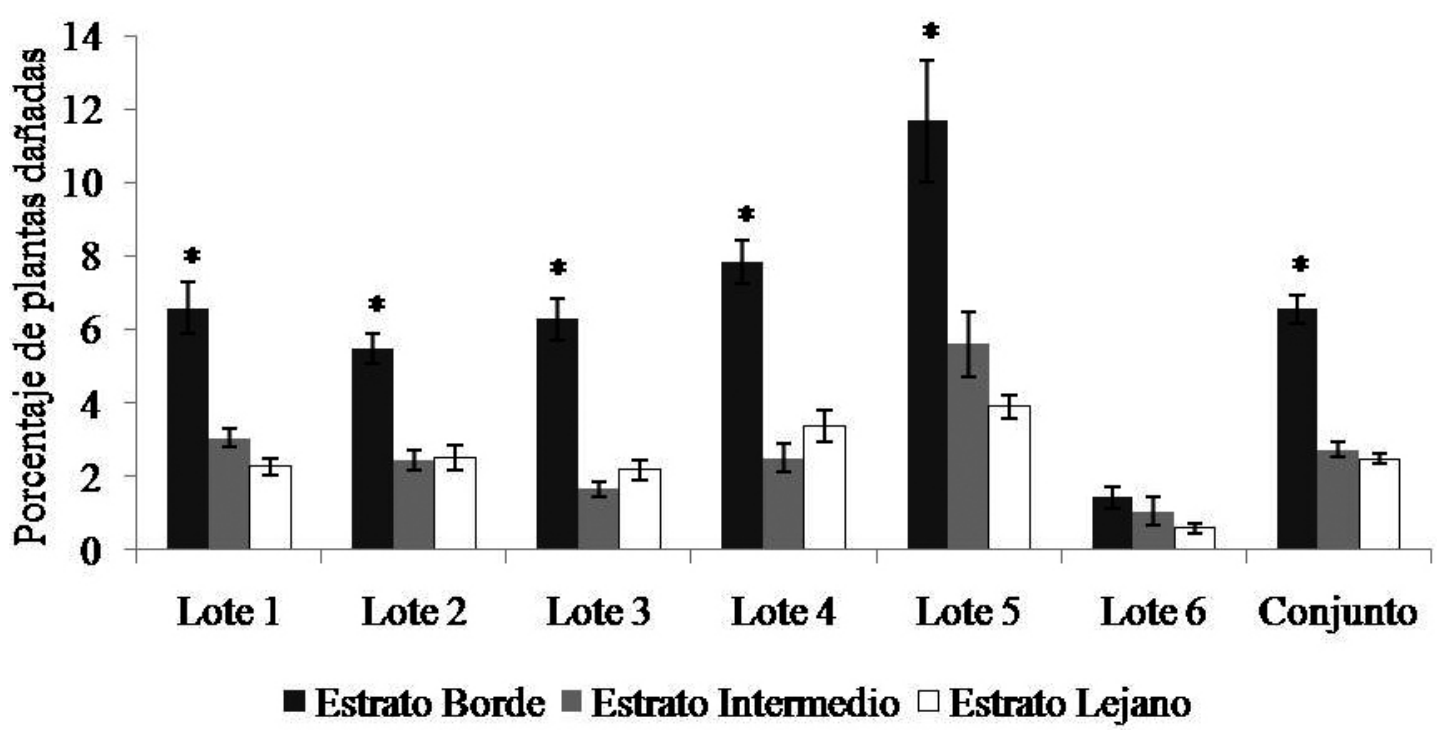

Figura 3. Modelo preliminar. Porcentaje promedio de plantas dañadas por lote y para el conjunto de datos por estratos con su desviación estándar. Los asteriscos indican diferencias significativas de porcentaje de daño en base al test no paramétrico de Kruskal-Wallis $(\mathrm{P}<0,05)$ corregido por Bonferroni.

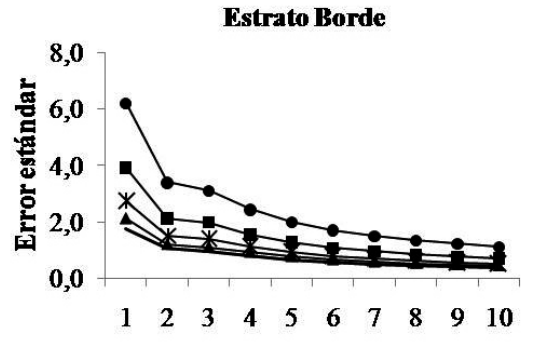

$\rightarrow 1$ submuestra $\quad \rightarrow-2$ submuestras

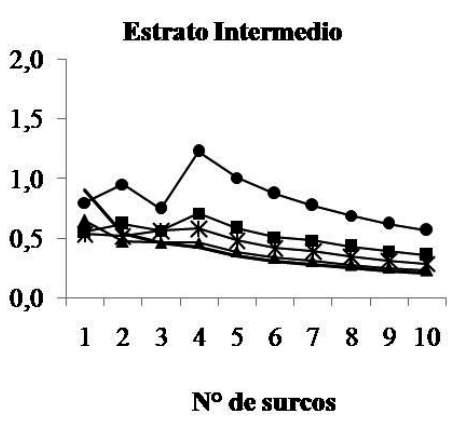

* 3 submuestras $\quad-4$ submuestras
Estrato Lejano

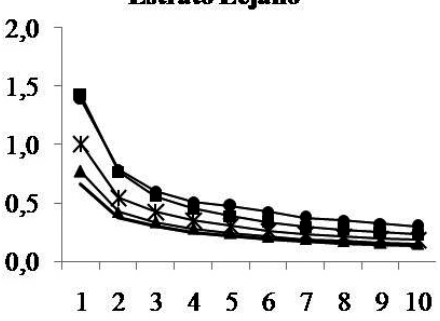

-5 submuestras

Figura 4. Variación del error estándar al ir aumentando el número de surcos, considerando de 1 a 5 submuestras por surco, para el conjunto de datos por estrato.

(Figura 5). El porcentaje promedio de plantas dañadas por aves en cada uno de los ocho lotes evaluados con el modelo definitivo varió entre $2 \%$ y $49 \%$ en el estrato de alta probabilidad de daño, y de $0,3 \%$ a $9,3 \%$ en el de baja probabilidad de daño (Figura 6). Los relevamientos llevaron entre 12 y 53 minutos para el estrato de alta probabilidad de daño, y entre 9 y 17 minutos para el de baja probabilidad de daño, totalizando entre 21 y 70 minutos para el muestreo de un lote. La evaluación de cada submuestra tomó entre 1 y 5 minutos, dependiendo del nivel de daño y la visibilidad de las plántulas dada por el tipo de rastrojo.
Se comprobó, por ambos modelos, que el daño por aves en lotes de soja recién emergida no es espacialmente homogéneo sino que es mayor en los sectores del lote próximos a vegetación arbórea. Este patrón de daño ha sido observado en otros cultivos como trigo, sorgo, girasol y vid (Somers et al., 2002) y también en otros grupos como mamíferos (Retamosa et al., 2008). Si bien la evaluación del daño por aves en este estudio se basó fundamentalmente en palomas, por ser las únicas aves de mediano porte que se alimentan de este cultivo en Uruguay, el método se podría utilizar para evaluar daño por otras especies de aves con características similares en tamaño y 
comportamiento alimenticio asociado al refugio que brindan los montes. Cabe mencionar que, si bien no fue evaluado, se observaron otros elementos del paisaje, como alambrados o postes, que también podrían generar un efecto similar al de los árboles, dado que las aves suelen utilizarlos como perchas y descienden desde allí al cultivo para alimentase. La

2 estratos por lote:

A-Estrato de alta probabilidad de daño

B-Estrato de baja probabilidad de daño

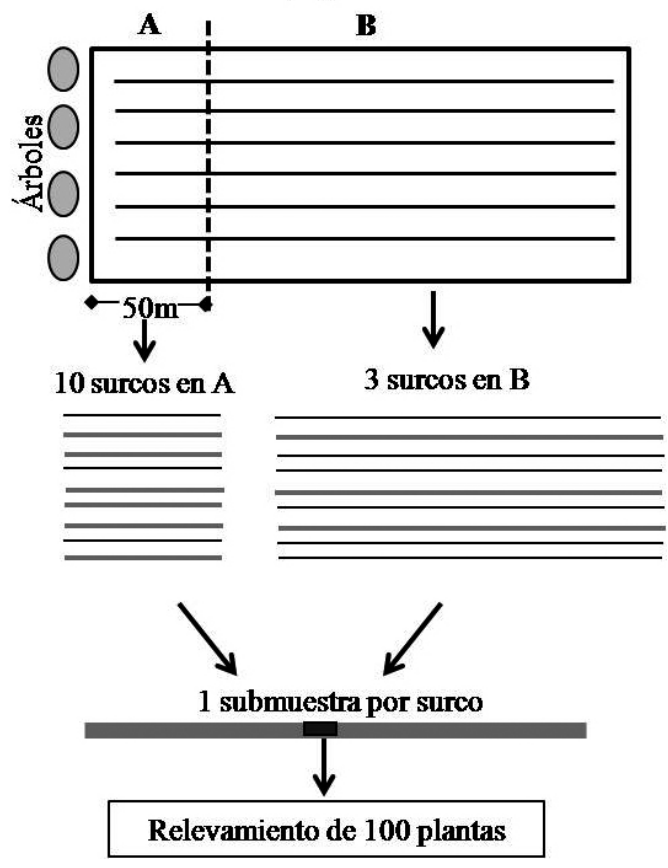

Figura 5. Esquema del modelo definitivo usado para evaluar el daño por aves en soja recién emergida. magnitud del daño en las zonas cercanas a árboles fue menor al 10\% de plantas dañadas en la mayoría de los casos, pudiendo alcanzar; sin embargo, valores cercanos al 50\%. El estrato de baja probabilidad de daño presentó valores generalmente menores al $1 \%$ de plantas dañadas.

La determinación de los estratos y la correcta identificación del daño generado por aves, son dos aspectos claves en la aplicación correcta del modelo de evaluación de daño propuesto. En especial, identificar correctamente el estrato de alta probabilidad de daño, es fundamental para obtener información que sea de utilidad para la toma de decisiones. Por ello, se recomienda realizar un recorrido previo del lote para identificar si existe una zona aledaña a árboles en la que, a simple vista, se observe mayor cantidad de plantas dañadas, y allí ubicar el estrato de mayor probabilidad de daño. Del mismo modo, es crucial identificar de forma correcta el factor causal del daño observado, dado que existen otros agentes que también dañan la soja además de las aves, tanto biológicos (varias especies de artrópodos como isópodos e insectos, mamíferos) como mecánico, producido por factores abióticos como viento, granizo y suelo compacto (Saluso et al., 2006; Retamosa et al., 2008; Ribeiro et al., 2008).

El método de muestreo de daño presentado aquí es específico para estimar el daño ocasionado por aves, ya que toma en cuenta el patrón espacial de distribución del daño que estas generan en el cultivo de acuerdo a su comportamiento. Por este motivo no sería aplicable, en primera instancia, al daño generado por otro tipo de animales. A fin de contar

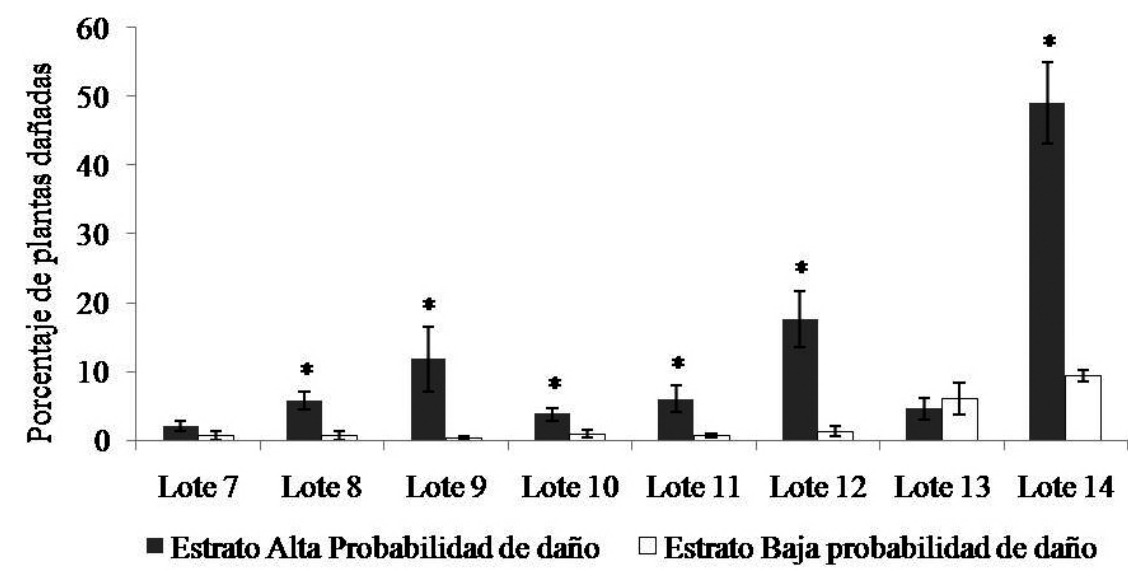

Figura 6. Modelo definitivo. Porcentaje promedio de plantas dañadas por lote con su desviación estándar. Los asteriscos indican diferencias significativas de porcentaje de daño en base al test no paramétrico de Mann Whitney $(\mathrm{P}<0,05)$. 
con evaluaciones confiables del daño ocasionado por aves en lotes de soja, y poder orientar de manera más objetiva las decisiones de manejo, sería importante ampliar el muestreo en el futuro abarcando distintas situaciones y condiciones ambientales.

\section{Conclusiones}

Se generó un método de evaluación de daño por aves que considera la heterogeneidad espacial en el patrón de daño que estas producen, a la vez que minimiza el esfuerzo de muestreo sin perder validez estadística. Debido a que este método implica la identificación de estratos de alta y baja probabilidad de daño, la implementación del modelo desarrollado permite un tratamiento diferencial entre estratos. Es decir, si el daño difiere significativamente entre estratos y es alto solo en el estrato de alta probabilidad, puede bastar aplicar una medida de protección del cultivo o decidir resembrar solo en dicho estrato y no en todo el lote, ahorrando al productor tiempo y dinero.

\section{Agradecimientos}

Al Instituto Nacional de Investigación Agropecuaria (INIA) La Estanzuela por su apoyo técnico y económico. A la Mesa Tecnológica de Oleaginosas (MTO) y a la Cooperativa Agraria Nacional (COPAGRAN) que facilitaron gran parte de los lotes de soja. A la Agencia Nacional de Investigación e Innovación (ANII) por su ayuda económica mediante dos becas de iniciación, códigos INI_X_2011_1_3782 e INI_X_2011_1_3805. A la Dirección General de Servicios Agrícolas. Al Téc. Ag. Wilfredo Ibáñez por su asistencia con los análisis estadísticos. A los productores y técnicos de cada predio por su colaboración.

\section{Literatura Citada}

Calamari, N.C.; Dardanelli, S.; Canavelli, S.B.

2011. Variaciones en la abundancia poblacional de palomas medianas a lo largo del tiempo. En: Bases para disminuir el daño por palomas en cultivos extensivos. Estación Experimental Agropecuaria Paraná. Ediciones INTA Paraná, Argentina, pp. 23-28.

Calamari, N.C.; Dardanelli, S.; Canavelli. S.B.

2012. Variaciones en la abundancia de palomas y cotorras en la región pampeana. En: Jorn. Actual. Técnica en Sorgo, Maíz y Girasol. Lallana, V. (Ed.). Ediciones INTA Paraná. Paraná, Argentina, pp. 139-146.

Calvi, C.; Besser, J.F.; De Grazio, J.W.

1976. Protecting Uruguayan Crops From. Proc. Seventh Bird Control Semin., (81): 255-258.

Catacora-Vargas, G.; Galeano, P.; Agapito-Tenfen, S.Z.; Aranda, D.; Palau, T.; Nodari, R.O.

2012. Producción de Soya en el Cono Sur de las Américas: Actualización Sobre el Uso de Tierras y Pesticidas. Virmegraf. Cochabamba, Bolivia, $50 \mathrm{p}$.

Coulter, J.A.; Nafziger, E.D.

2008. Yield and agronomic responses of soybean to cotyledon and unifoliolate node removal. Crop Sci 48 (1): 353-356.

Dardanelli, S.; Calamari, N.C.; Beatriz, C.S.; Zaccagnini, M.E. 2011. Biología de la paloma mediana (Zenaida auriculata), manchada (Patagioenas maculosa) y picazuro (Patagioenas picazuro). En: Bases para disminuir el daño por palomas en cultivos extensivos. Serie Exte. EEA Paraná. Paraná, Argentina, pp. 11-22.

Dirección de Climatología y Documentación, D.N. 2014. Informe técnico de la Dirección Nacional de Meteorologíale. INUMET. Disponible en: http://www. meteorologia.com.uy/ServCli/ caracteristicasEstacionales. Consultado el 02/08/2016.
Dolbeer, R.A.

1981. Cost-Benefit Determination of Blackbird Damage Control for Cornfields. Wildl. Soc. Bull. 9 (1): 44-51.

Dolbeer, R.A.; Holler, N.R.; Hawthorne, D.W.

1994. Identification and assessment of wildlife damage: an overview. En: The Handbook: Prevention and Control of Wildlife Damage. Hygnstrom, S.E., Timm, R.M., Larson, G.E. (eds.). University of Nebraska-Lincoln. Nebraska, United States of America, pp. A1-A18.

Engeman, R.M.

2000. Economic considerations of damage assessment. En: Proceedings of the Third National Wildlife Research Centre Special Symposium, Fort Collins, Co. USDA National Wildlife Research Center Symposia, pp. 36-41.

Hammer, Ø.; Harper, D.A.T.; Ryan, P.D.

2001. Paleontological statistics software package for education and data analysis. Palaeontol. Electron 4 (1): 9-18.

Moscardi, F.; Bueno, A.; Bueno, R.; García, A.

2012. Soybean response to different injury levels at early developmental stages. Ciência Rural 42 (3): 389-394.

Olivera, L.; Rodríguez, E.

2014. Aumentando rendimiento cultivos extensivos disminuyendo daño de aves. Serie FPTA No 56 . Hemisferio Sur S.R.L. Montevideo, Uruguay, 47 p.

Olson, D.M.; Dinerstein, E.; Wikramanayake, E.D.; Burgess, N.D.; Powell, G.V.N.; Underwood, J.E.C.; D’amico, A.; Itoua, I.; Strand, H.E.; Morrison, J.C.; Loucks, C.J.; Allnutt, T.F.; Ricketts, T.H.; Kura, Y.; Lamoreux, J.F.; Wettengel, W.W; Hedao, P.; Kassem, K.R. 2001. Terrestrial Ecoregions of the World: A New Map of Life on Earth. Bioscience 51 (11): 933.

Otis, D.L.

1992. Recommendations on the need and use of statistical surveys for evaluating the impact of bird damage to crops 
in Uruguay and Argentina. Unpubl. report prepared for the United Nations Food and Agriculture Organization, January 27, 1992. U.S. Fish and Wildlife Service, South Carolina Cooperative Research Unit, Clemson Univ, Clemson, South Carolina, USA. 34 pp.

Reolón, L.; Iturburu, M.; Hoffmeister, M. (Eds).

2015. Informe del Estado del Ambiente de Uruguay. Primera. MVOTMA, Montevideo, Uruguay, $277 \mathrm{pp}$.

Retamosa, M.I.; Humberg, L.A.; Beasley, J.C.; Rhodes. O.E. 2008. Modeling wildlife damage to crops in northern Indiana. Human-wildlife Conflicts, 2 (2): 225-239.

Ribeiro, A.F.; Castiglioni, E.; Silva, H.

2008. Insectos de la soja en Uruguay: Manual ilustrado de reconocimiento de plagas y enemigos naturales. Hemisferio Sur. Montevideo, Uruguay, 82 pp.

Rocha, P.J.; Villalobos,V.M.

2012. Estudio comparativo entre el cultivo de soja genéticamente modificada y el convencional. Ministerio de Ganaderia, Agricultura y Pesca de Argentina (MGAP, AR) e Instituto Interamericano de Cooperación para la Agricultura (IICA). San José, Costa Rica, 88 p.

Rodríguez, E.; Tiscornia, G.; Olivera, L.

2011. Disminución del daño por aves en pequeños predios. Estrategia de disminución del daño por aves en pequeños predios de alto valor utilizando métodos no contaminantes. Serie FPTA N ${ }^{\circ}$ 29, Montevideo, Uruguay, 64 pp.
Rodríguez, E.; Zaccagnini, M.E.

1998. Manual de capacitación sobre manejo integrado de aves perjudiciales a la agricultura (E.N. Rodríguez and M.E. Zaccagnini, Eds.). MGAP, FAO, Ministerio de Economía y Obras y Servicios Públicos Servicio Nacional Sanidad y Calidad Agroalimentaria, Montevideo, Uruguay, 172 pp.

Saluso, A.; De Carli, R.; Zaccagnini, M.E.; Decarre, J.; Cáceres. C. 2006. Guía práctica para el control quimico de artrópodos plaga en soja considerando el riesgo de toxicidad aguda para las aves. Ediciones INTA. Entre Ríos, Argentina, $20 \mathrm{pp}$.

Somers, C.M.; R.D. Morris; C.M. Somers; and Morris, R.D. 2002. Birds and Wine Grapes: Foraging Activity Causes Small-Scale Damage Patterns in Single Vineyards. J. Appl. Ecol. 39 (3): 511-523.

USDA

2015. World Agricultural Supply and Demand Estimates. WASDE 535. Disponible en: http://www.usda.gov/oce/ commodity/wasde/latest.pdf. Conusltado el 02/08/2016.

Zaccagnini, M.E.

1998. Evaluación de daños. En: Manual de Capacitación sobre Manejo Integrado de Aves Perjudiciales a la Agricultura. Rodríguez, E.N.; Zaccagnini, M.E. (eds.), MGAP, FAO, Ministerio de Economía y Obras y Servicios Públicos Servicio Nacional Sanidad y Calidad Agroalimentaria, Montevideo, Uruguay, pp. 85-116. 